\title{
OBSERVATIONS
}

\section{Existence de Rickettsia canis au Tchad}

\author{
par P. RECEVEUR et G. HUGAUD
}

CETTE affection, soupçonnée depuis longtemps au Tchad, vient d'être confirmée en août 1948 dans les conditions suivantes :

Le Docteur Vétérinaire Hugaud, venant au Tchad, avait apporté avec lui une jeune chienne fox, âgée de 3 ans. Arrivée à FortLamy le 11 août, cette chienne a commencé unẹ quinzaine de jours après à présenter une fièvre marquée, accompagnée de refus de nourriture et de somnolence. Comme elle était porteur de nombreuses tiques, du genre Rhipicephalus, et bien qu'aucune hémoglobinurie ne se soit manifestée, il parut utile de suspecter la piroplamose. Aussi fut-il effectué un frottis sanguin le 31 août, colore et examiné le lendemain. Rien d'anormal ne fut décélé. Comme, d'autre part, les symptômes s'amendaient, aucun traitement ne fut entrepris. Cet amendement fut de courte durée : une reprise motivait un deuxieme examen le 6 septembre qui, comme le précédent, n'apporta aucun éclaircissement. A ce moment on notait déjà une certaine parésie du traín postérieur, en même temps que la somnolence s'accroissait, avec perte totale d'appétit.' Un traitement symptomatique était appliqué.

Entre temps, du 12 au 20 septembre, noug étions obligés de nous absenter de Larny et, le 2l, à notre retour, nous trouvions l'animal on mauvais état, les symptomes nervøux (paresse, somnolence) s'étant encore accusés.

Le 22, nous effectuions un nouveau frottis sanguin, en même temps que nous faisions une injection de trypan bleu, suspectant toujours une piroplasmose possible, chose très courante sur les animaux importés, et ceci bien que nous n'ayions constaté aucun symptôme d'hémoglobinurie. I'examen du frottis, après coloration au Giemsa lent, nous a permis de trouver le 25 au matin des Rickettsia intraleucocytaires, répondant aux descriptions types, les globules parasités étant d'ailleurs peu nombreux. Par contre, malgré des recherches méthodiquement poursuivies pendant fort longtemps, aucun piroplasme n'a $\mathrm{F}_{\mathrm{L}} \mathrm{u}$ être mis en évidence.

La mort de l'animal est survenue dans la soirée.

Cette chienne s'est donc comportée comme un animal neuf et nous a permis de décéler la Rickettsiose canine au Tchad.

Notons que la symptomatologie de l'affection s'est révélée absolument superposable à celie décrite classiquement. $A$ aucun moment nous n'avons rencontré les symptômes spéciaux décrits par Girard et Rousselot en A.O.F. et considérés par cés derniers comme pathognomoniques (symptôme de l'oreille) et ce, malgré la similitude des climats, les rapports certains existant entre I'Á.O.F. et I'A.E.F. et le fait d'avoir notre attention attirée sur ce point. Nous pensons donc - quant à nous - que l'on doive s'en tenir pour le Tchad à la description classique de l'affection.

\section{Un cas de fibromatose cervicale diffuse chez le Chameau}

\section{par A. BOUÉ, vétérinaire lieutenant}

UNE chamelle de 10 ans nous est présentée pour être livrée à la boucherie. A la base du cou, à droite, on remarquait deux masses dures, non sensibles, adhèrentes à la peau, l'une antérieure en position du volume d'un cuf de dinde, l'autre postérieure de la grosseur du poing. L'animal est sacrifié le même jour.

A l'autopsie, on trouve :

10 un envahissement du tissu conjonctif de toute la face droite de l'encolure par des lames fibreuses de la $3^{e}$ à la $6^{\text {e }}$ cervicale, constituant en quelque sorte une gangue fibreuse (voir dessin ci-joint);

$2^{\circ}$ également à droite, une plaque fibreuse large de 12 centimètres, très épaisse (1-2 cm.) allant de l'apophyse transverse de la $3^{\text {e }}$ cervicale à celle de la $5 \mathrm{e}$, adhérente à la peau et au plan musculaire sous-jacent;

$3^{0}$ à la base du cou, de ce même côté, deux masses

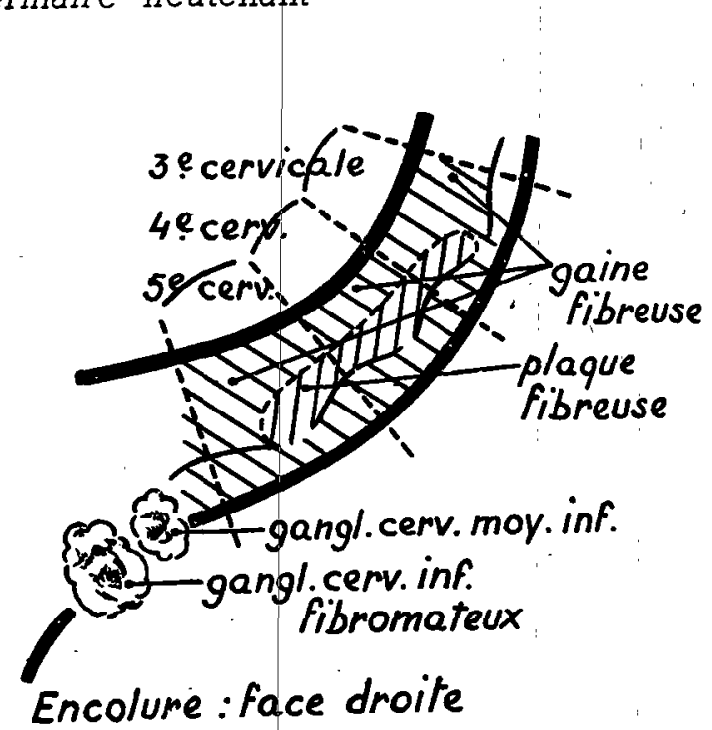


ganglionnaires hypertrophiées, encapsulées par du tissu fibrolardacé!' Il s'agit du'ganglion cervical moyen inférieur (G. Petit) et du ganglion cervical inférieur (G. Petit) encore dénommé prépectoral (Leese). A la coupe, le tissu lymphatique est onscrré dans des mailles de tissu fibreux.

L'examen des autres régions ne présentait rien de particulier. Nous avions déjà signalé ailleurs. (Archives de I'Institut Pasteur d'Algérie, t. XXIII, $\mathrm{n}^{0}$ 4) une fibromatose des membres postérieurs. Dans les deux cas; les ganglions lymphatiques ont réagi et ont été trouvés fibromateux; ceci vérifierait la théorie admettant la voie lymphatique pour Ic processus cancérigène. L'origine de cette fibromatose n'est toutefois pas solutionnée.

\title{
Quelques anomalies dentaires chez le Chameau
}

\author{
par A. BOUÉ, vétérinaire lieutenant
}

ЦES anomalies dentaires notées ci-dessous ressortent de l'observalion systémalique de 503 drumadaires adultes abattus à l'abattoir de C.B.

Nous envisagerons d'abord les anomalies des arcades dentaires puis celle's des dents.

\section{I. - ANOMALTES DES ARCADES DENTAIRES.}

Elles affectent l'arcade incisive inférieure; on sait qu'au maxillaire supérieur ne subsistent que les coins. Elles corriespondont à un manque de symćtrie tant par rapport au plan axial que par rapport au plan horizontal.

a) Asymétrie par rapport au plan axial. - Trois cas observés. L'arc alvéolaire normalement parabolique est ici asymétrique; alors qu'un côté est normal, l'autre, moins incurvé, tend vers la ligne droite; les incisives sont de ce fait en position rejetées en arrière. Le décalage des dents influe sur la position des canines et dès prémolaires caniniformes qui ne se correspondent plus. Les incisives en retrait sont plus usées que celles du côté normal. Voir schéma $n^{\circ} 1$.

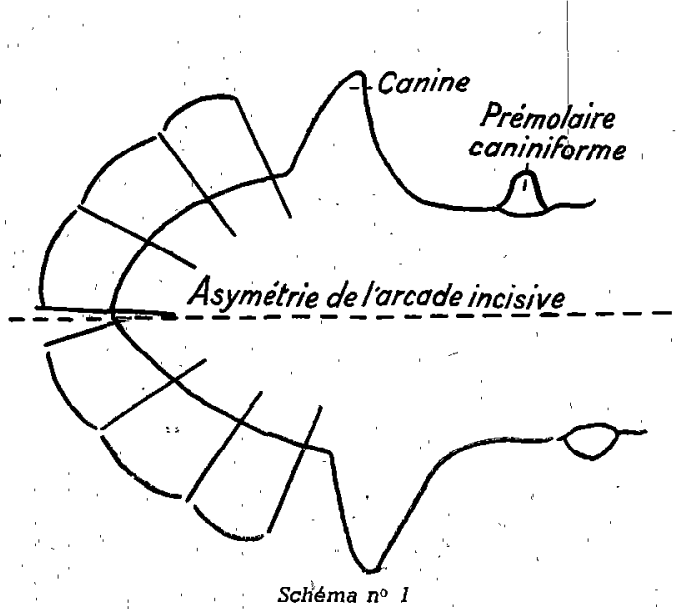

b) Asymétrie par rapport au plan horizontal. L'asymétrie est 'due à une legere torsion de l'extrémité du maxillaire. Si la table dentaire reste horizontale par l'usure, la table comparée de deux incisives homologues n'offre pas une forme identique, les dents'n'ayant pas la même longueur. Deux. cas observés.

\section{II. - ANOMALIES DES DENTS.}

Celles qui intéressent les incisives procèdent $\alpha$ 'une direction et d'une implantation vicieuses; elles sont rares. Les variations numériques, par contre, concernent les prémolaires et les molaires.

a) Anomalie de direction. - Une seule observation constatée sur un coin gauche inférieur. Lia dent présente un mouvement de torsion autour de son axe; redressée, elle ne participe pas à la formation de l'arcade incisive et se dirige à l'intérieur de la bouche. Voir dessin no 2 .

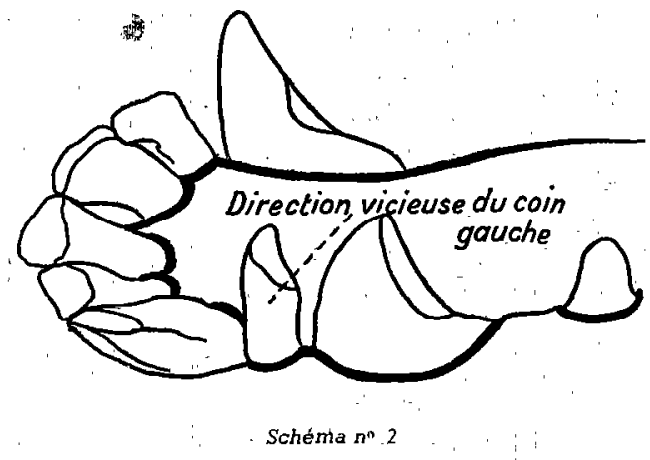

b) Anomalie d'implantation. - Une seule observation également : la pince gauche est implantée à l'intérieur de l'arcade dentaire. Par suite d'un diastème entre la mitoyenne et le coin droit, cottc mitoyenne vient occuper la place de la pince droite 